\title{
Analytical Solutions of Moisture Flow Equations and Their Numerical Evaluation
}

\author{
A. G. Gibbs
}

April 1981

Prepared for the U.S. Department of Energy under Contract DE-AC06-76RLO 1830

Pacific Northwest Laboratory

Operated for the U.S. Department of Energy by Battelle Memorial Institute 


\title{
NOTICE
}

This report was prepared as an account of work sponsored by the United States Government. Neither the United States nor the Deparment of Energy, nor any of their employees, nor any of their contractors, subcontractors, or their employees, makes any warranty, express or implied, or assumes any legal liability or responsibility for the accuracy, completeness or usefulness of any information, apparatus, product or process disclosed, or represents that its use would not infringe privately owned rights.

The views, opinions and conclusions contained in this report are those of the contractor and do not necessarily represent those of the United States Government or the United States Department of Energy.

\author{
PACIFIC NORTHWEST LABORATORY \\ operated by \\ BATTELLE \\ for the \\ UNITED STATES DEPARTMENT OF ENERGY \\ Under Contract DE-AC06-76RLO 1830
}

\author{
Printed in the United States of America \\ Available from \\ National Technical Information Service \\ United States Department of Commerce \\ 5285 Port Royal Road \\ Springfield, Virginia 22151
}

Price: Printed Copy $\$$

$*$; Microfiche $\$ 3.00$

$\begin{array}{cc}\text { *Pages } & \text { NTIS } \\ \text { Selling Price } \\ 001-025 & \$ 4.00 \\ 026-050 & \$ 4.50 \\ 051-075 & \$ 5.25 \\ 076-100 & \$ 6.00 \\ 101-125 & \$ 6.50 \\ 126-150 & \$ 7.25 \\ 151-175 & \$ 8.00 \\ 176-200 & \$ 9.00 \\ 201-225 & \$ 9.25 \\ 226-250 & \$ 9.50 \\ 251-275 & \$ 10.75 \\ 276-300 & \$ 11.00\end{array}$


PNL -3680

UC -70

\section{2}

ANALYTICAL SOLUTIONS OF MOISTURE FLOW EQUATIONS AND THEIR NUMERICAL EVALUATION

A. G. Gibbs

Apri1 1981

Prepared for the U.S. Departinent of Energy under Contract DE-AC06-76RLO 1830

Pacific Northwest Laboratory Richland, Washington 99352 


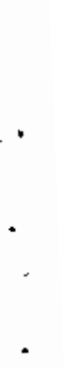




\section{ABSTRACT}

The role of analytical solutions of idealized moisture flow problems is discussed. Some different formulations of the moisture flow problem are reviewed. A number of different analytical solutions are summarized, inciuding the case of idealized coupled moisture and heat flow. The evaluation of special functions which commoniy arise in analytical solutions is discussed, including some pitfalls in the evaluation of expressions involving combinations of special functions. Finally, perturbation theory methods are sumarized which can be used to obtain good approximate analytical solutions to problems which are too complicated to solve exactly, but which are close to an analytically solvable problem. 


\section{CONTENTS}

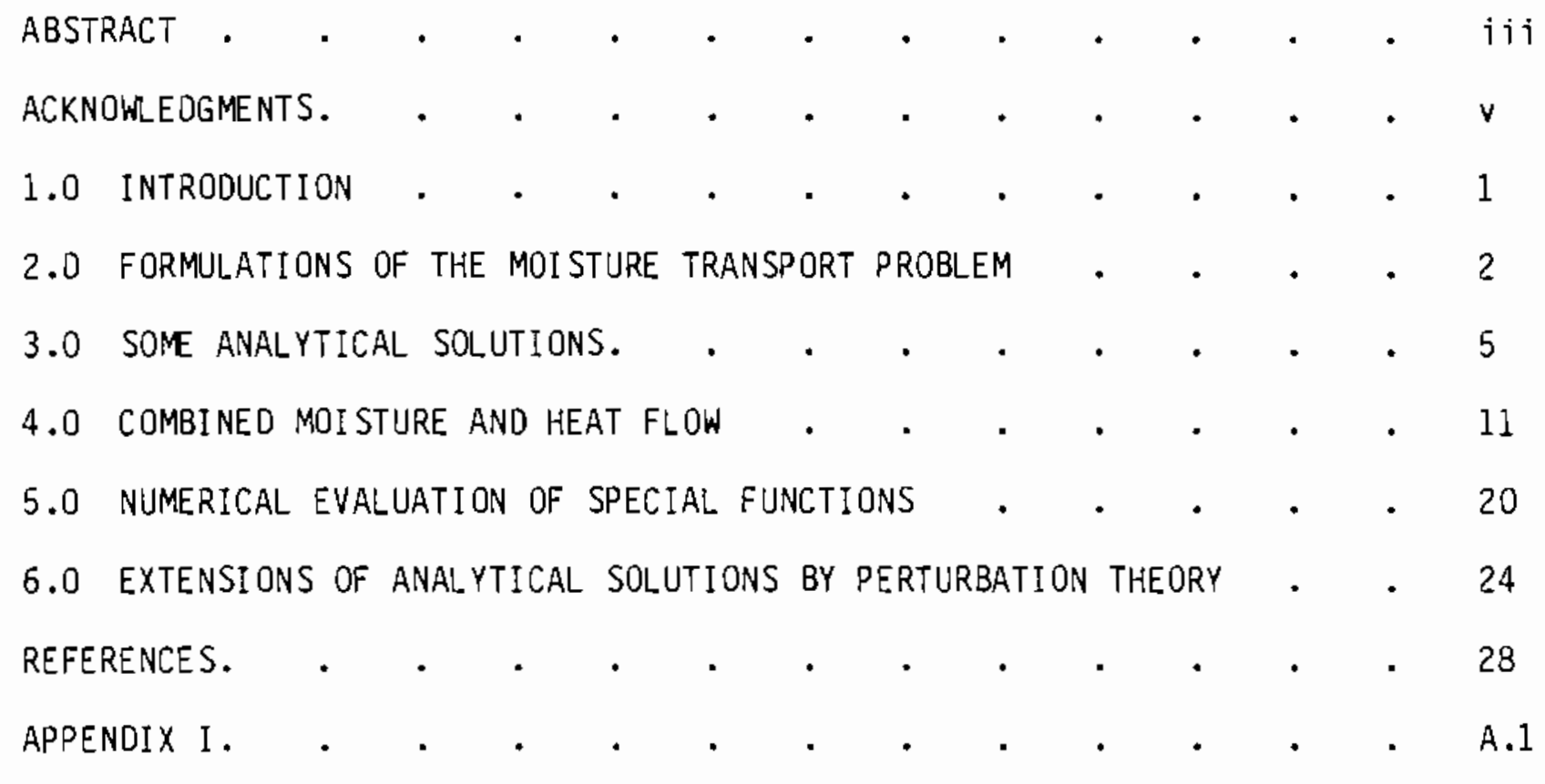




\section{ACKNOWLEDGMENTS}

This project was jointly sponsored by Pacific Northwest Laboratory and Rockwell Hanford Operations. 


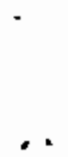

. 


\subsection{INTROOUCTION}

The most general formulations of the moisture flow problem lead to partial differential equations which are nonlinear and which have variable coefficients. These equations are not solvable by known analytical methods, but must be treated numerically, using finite differences, finite elements, collocation methods, etc. It is only with considerable simplification that transport problems sufficiently idealized to be analytically tractable are obtained.

Different philosophies can be advanced to justify the seeking of such solutions. Clearly, whatever the ir physical shortcomings, analytical solutions can provide important test cases for more general numerical methods. In a given application, to the extent that essential physics is retained, the form of analytical solutions may also provide important insights into the behavior of solutions of real-world problems, and may thus help in the design of numerical methods. Also if, in a particular application, only small changes in a solution from its initial values are anticipated, then a linearization of the general equations about those intial values may lead to a realistic set of equations which are simple enough to treat analytically. Finally, some problems too difficult to solve exactly are "close enough" to solvable problems so that approximate analytical solutions may be obtained by perturbation methods. 


\subsection{FORMULATIONS OF THE MOISTURE TRANSPORT PROBLEM}

Various levels of generality are possible in formulating moisture flow problems. Quite generally, the moisture content $\theta$ satisfies the equation:

$$
\frac{\partial \theta}{\partial t}=-\nabla \cdot q_{\theta}+S_{\theta}
$$

where $q_{\theta}$ denotes the moisture flux and $S_{\theta}$ any local sources of moisture. For the case of isotropic soils, assuming Darcy's law to be valid, the flux $\mathrm{q}_{\theta}$ can be written as:

$$
q_{\theta}=-K(\nabla \Psi-\hat{z})
$$

where $K$ is the hydraulic conductivity, $\psi$ the moisture potential, and $\hat{z}$ a unit vector in the downward direction (representing the effect of gravity). Using (2.2) in (2.1) gives:

$$
\frac{\partial \theta}{\partial t}=\nabla \cdot K \nabla \Psi-\frac{\partial K}{\partial z}+S_{\theta}
$$

If $\Psi$ is determined as a unique function of $\theta$ (empirically), then by defining the soil water diffusivity:

$$
D(\theta)=K \frac{d \Psi(\theta)}{d \theta},
$$

Equation (2.3) can be rewritten as:

$$
\frac{\partial \theta}{\partial t}=\nabla \cdot D(\theta) \nabla \theta-\frac{d K(\theta)}{d \theta} \frac{\partial \theta}{\partial z}+S_{\theta}
$$

In genera], $D(\theta)$ and $K(\theta)$ are complicated functions of $\theta$. 
Alternatively, the moisture potential $\psi$ may be treated as the dependent variable rather than $\theta$. Then, defining:

$$
C(\Psi)=\frac{d \theta}{d \Psi}=\frac{1}{\partial \Psi / d \theta}
$$

allows (2.3) to be written as:

$$
C(\psi) \frac{\partial \psi}{\partial t}=\nabla \cdot K(\Psi) \nabla \psi-\frac{d K(\psi)}{d \psi} \frac{\partial \psi}{\partial z}+S_{\theta}
$$

A third variant is obtained by applying a Kirchoff transformation to Equation (2.7). Define a new independent variable $\phi(\psi)$ by:

$$
\phi(\Psi)=\int_{\Psi_{0}}^{\Psi} K\left(\Psi^{\prime}\right) d \Psi^{\prime}
$$

where $\psi_{0}$ is some constant. (The function $\phi$ is known as the matric flux potential.) Then, clearly:

$$
\nabla \phi=\frac{d \phi}{d \Psi} \nabla \Psi=K(\Psi) \nabla \Psi
$$

Thus, Equation (2.7) becomes:

$$
S_{\theta}+\nabla^{2} \phi-\frac{d K}{d \Psi} \frac{\partial \Psi}{\partial z}=C(\Psi) \frac{\partial \psi}{\partial t}
$$

and by defining:

$$
\frac{d \theta}{d \phi}=B(\phi)=C(\Psi) \frac{d \psi}{d \phi}=\frac{C(\Psi)}{d \phi / \partial \psi}=\frac{C(\Psi)}{K(\Psi)}
$$


Equation (2.10) becomes:

$$
B(\phi) \frac{\partial \phi}{\partial t}=\nabla^{2} \phi-\frac{d K(\phi)}{d \phi} \frac{\partial \phi}{\partial z}+S_{\theta}
$$

In order to obtain an analytical solution to any of the above equations, some simple forms must be assumed for $D(\theta)$ and $K(\theta), C(\psi)$ and $K(\psi)$, or $B(\phi)$ and $K(\phi)$. Also, an elementary geometry and simple boundary conditions are required.

Boundary conditions usually state that either $\theta$ or $\psi$ is known on part of the boundary, and that the moisture flux $a_{\theta}$ is known on the remaining part of the boundary. If the p-formulation of Equation (2.12) is being used, then specifying $\psi$ on the boundary is equivalent, via Equation (2.8), to specifying $\phi$, while specifying $q_{\theta}$ is, via Equations (2.2) and (2.9), equivalent to specifying $\nabla \phi$, since:

$$
q_{\theta}=\nabla \phi+k \hat{z}
$$




\subsection{SOME ANALYTICAL SOLUTIONS}

The most elementary solutions of the equations of the previous section are obtained by assuming that coefficients, such as $D(\theta)$, are constant, and prescribing constant initial and boundary conditions. Solutions of the resulting constant-coefficient partial differential equations may be obtained by a variety of methods. When boundary and initial conditions are compatible, similiarity solutions may exist (Hansen 1964). These are solutions in which the independent variables occur only in particular combinations so the solution actually depends on a smaller number of variables. One such example occurs when $\theta(z, t)=\theta(z / \sqrt{t})$. The transformation to the new variable $z / \sqrt{t}$ is known as Boltzmann's transformation, and leads to a simpler equation for $\theta$. However, such similarity solutions are rather special and occur only for certain combinations of initial and boundary conditions. More generally, the partial differential equations must be solved by the method of separation of variables (Rainville 1958), or by using integral transforms (Tranter 1974). The simplest problem of all is the "horizontal infiltration problem" in which the z-variation does not occur. Then, with a constant $D$ and no sources, Equation (2.5) reduces to:

$$
\frac{\partial \theta}{\partial t}=D \frac{\partial^{2} \theta}{\partial x^{2}}
$$

With the elementary boundary and initial conditions:

$$
\begin{array}{ll}
\theta(x, 0)=\theta_{j} & x>0 \\
\theta(0, t)=\theta_{u} & t>0
\end{array}
$$

the solution, due to Philip (1966), is:

$$
\theta=\theta_{i}+\left(\theta_{u}-\theta_{i}\right) \operatorname{erfc} \frac{x}{2 \sqrt{D t}}
$$


(The above problem is, of course, an exact analog of a problem in heat conduction. Many other heat conduction problems are mathematically equivalent to idealized forms of one of the formulations of the moisture equations of the previous section. Thus, references such as Carslaw and Jaeger (1948) can be browsed for analytical solutions of problems that may also be of use in moisture flow applications.)

Another idealized problem is the "vertical infiltration problem" in which the solution depends only on the $z$-coordinate. Then, Equation $(2.5)$, with 0 and $d K / d \theta=X_{\theta}$ assumed constant, becomes:

$$
\frac{\partial \theta}{\partial t}=\frac{\partial^{2} \theta}{\partial z^{2}}-x_{\theta} \frac{\partial \theta}{\partial z}
$$

Subject to the conditions:

$$
\begin{array}{ll}
\theta(z, 0)=\theta_{i} & z>0 \\
\theta(0, t)=\theta_{u} & t>0
\end{array}
$$

the solution obtained by Philip (1966), is:

$$
\begin{aligned}
\theta & =\theta_{i}+\frac{\theta_{u}-\theta_{i}}{2}\left[\operatorname{erfc}\left(\frac{z-k_{\theta} t}{2 \sqrt{D t}}\right)\right. \\
& \left.+\exp \left(k_{\theta} z / 0\right) \operatorname{erfc}\left(\frac{z^{+k_{\theta} t}}{2 \sqrt{D t}}\right)\right]
\end{aligned}
$$

An analogous solution was obtained by Philip for the case of a spherically symetric problem with infiltration from a spherical cavity, but neglecting the gravity term (Philip 1969).

In a series of papers by warrick and his coworkers $(1974,1976,1978)$, analytical solutions are obtained for some simplified forms of the $\phi$-equation, i.e., Equation (2.12). They consider the hydraulic conductivity $k$ to be an exponential function of the moisture potential $\Psi$, i.e.: 


$$
K(\psi)=K_{0} e^{\alpha \psi}
$$

with $K_{0}$ and a constant. Then, with the constant $\psi_{0}$ taken to be $-\infty$, Equation (2.8) gives:

$$
\phi(\Psi)=\frac{K_{0}}{a} e^{\alpha \Psi}=\frac{1}{a} K(\Psi)
$$

Thus, for this model, the function $\phi$ is a constant multiple of $K$. In addition, the function $B(\phi)$, Equation (2.11), is taken to be a constant. (Since $B(\phi)=$ $\mathrm{d} \theta / \mathrm{d} \phi$, and since $\phi$ and $K$ are linearly related in the present case, then this is equivalent to assuming that $\mathrm{d} \theta / \mathrm{dK}$ is constant, or that $\mathrm{K}$ is linear as a function of 9 ). Using Equations (3.7) and (3.8) in Equation (2.12) simplifies it to:

$$
B \frac{\partial \phi}{\partial t}=\nabla^{2} \phi-\alpha \frac{\partial \phi}{\partial z}+S
$$

Solutions have been obtained for a number of different geometries and source/ sink distributions.

Warrick (1974) considered the case of a point source located at the origin on the surface of the ground. An initial condition:

$$
\phi(r, z \quad 0)=0
$$

was assumed, along with a boundary condition corresponding to no flow across the surface $z=0$ except at the origin. From Equations $(2: 13)$ and (3.7), this implies that:

$$
-\frac{\partial \phi}{\partial z}+a \phi=0 \quad z=0, r \neq 0, t>0
$$


The solution was obtained by superposing the solutions for point sources in an infinite medium. Dimensionless variables are defined by:

$$
R=\frac{a r}{2}, Z=\frac{\alpha z}{2}, T=\frac{a k t}{4}
$$

with:

$$
x=\frac{a}{B}
$$

Also, let:

$$
\rho=\sqrt{R^{2}+Z^{2}}
$$

Define the function:

$$
\begin{aligned}
\Phi_{B}(R, Z, T) & =\frac{e^{Z}}{2 \rho}\left\{e^{\rho} \operatorname{erfc}\left(\frac{\rho}{2 \sqrt{T}}+\sqrt{T}\right)\right. \\
& \left.+e^{-\rho} \operatorname{erfc}\left(\frac{\rho}{2 \sqrt{T}}-\sqrt{T}\right)\right\}
\end{aligned}
$$

Then the solution of Equation (3.9), subject to (3.10) and (3.11), can be written as:

$$
\begin{aligned}
\Phi & =\frac{8 \pi}{\alpha q} \phi=2\left\{\Phi_{B}(R, Z, T)\right. \\
& -\int_{Z}^{\infty} e^{\left.-2\left(Z^{\prime}-Z\right)_{\Phi_{B}}(R, Z, T) d Z^{\prime}\right\}}
\end{aligned}
$$

(The integral must be evaluated numerically.) Here, $q$ is the strength of the point source. 
Similar solutions were reported for line disk, and strip sources by Loman and Warrick $(1974,1976)$.

Solutions were obtained, also by Lomen and Warrick (1978), for the case of $2, t$ dependence only, but with an initial condition and a sink both dependent on depth, and for a time-dependent surface fiux. In terms of the dimensionless variables, $Z, T$, this problem is stated as:

$$
\frac{\partial \phi}{\partial T}=\frac{\partial^{2} \phi}{\partial Z^{2}}-2 \frac{\partial \phi}{\partial Z}-\frac{4}{a^{2}} S(Z)
$$

with:

$$
\begin{array}{ll}
\phi(Z, 0)=g(Z)+\phi_{\infty}, & T=0, Z>0 \\
-\frac{\partial \phi}{\partial Z}+2 \phi=\frac{2}{a} v(T), & T>0, Z=0
\end{array}
$$

Here, $g(Z)$ and $v(T)$ are given functions, and $\phi_{\infty}$ is a given constant.

The solution is written as:

$$
\phi(Z, T)=\phi_{\infty}+\phi_{S}(Z)+\phi_{T}(Z, T)
$$

where:

$$
\phi_{S}(Z)=\frac{U}{\alpha}-\frac{4}{a^{2}} \int_{Z}^{\infty} d Z^{\prime} e^{2\left(Z-Z^{\prime}\right)} \int_{0}^{Z^{\prime}} S(\xi) d \xi
$$

with:

$$
U=\frac{2}{a} \int_{0}^{\infty} S(Z) d z
$$


Also:

$$
\begin{aligned}
& \phi_{T}(Z, T)=2 \exp (Z-T) \int_{0}^{T}\left[\exp \left\{-Z^{2} / 4(T-\tau)\right\}\{\pi(T-\tau)\}-1 / 2\right. \\
& \left.-\exp (T-\tau+Z) \operatorname{erfc}\left\{Z / 2(T-\tau)^{1 / 2}+(T-\tau)^{1 / 2}\right\}\right] \\
& {\left[\{v(\tau)-U\} / a-\phi_{\infty}\right] \exp (\tau) d \tau+R\left(Z, T, g(Z)-\phi_{S}(Z)\right)}
\end{aligned}
$$

with:

$$
\begin{aligned}
& R(Z, T, f)=\exp (Z-T) \int_{0}^{\infty}(4 \pi T)^{-1 / 2}\left[\exp \left\{-\left(Z-Z^{\prime}\right)^{2} / 4 T\right\}+\right. \\
& \exp \left\{-\left(Z+Z^{\prime}\right)^{2} / 4 T\right\}-\exp \left(T+Z^{\prime} Z\right) \operatorname{erfc}\left\{\left(Z+Z^{\prime}\right) / 2 T^{1 / 2}\right. \\
& \left.\left.+T^{1 / 2}\right\}\right] f\left(Z^{\prime}\right) \exp \left(-Z^{\prime}\right) d Z^{\prime}
\end{aligned}
$$




\subsection{COMBINED MOISTURE AND HEAT FLOW}

Some analytical solutions are available for simplified forms of the coupled heat and moisture flow equations. One of these is due to Hauk (1971). He worked with equations for the temperature $T$ and the moisture potential $\Psi$. By appealing to the principles of irreversible thermodynamics he suggested that the appropriate form of the coupled equations is:

$$
\begin{aligned}
& C \frac{\partial T}{\partial t}=K_{a} \frac{\partial^{2} T}{\partial x^{2}}+w \frac{\partial^{2} \psi}{\partial x^{2}} \\
& C_{m} \frac{\partial \psi}{\partial t}=v \frac{\partial^{2} T}{\partial x^{2}}+k_{a} \frac{\partial^{2}}{\partial x^{2}}(\Psi-g x)
\end{aligned}
$$

Here $C, C_{m}, K_{a}, k_{a}, \vee w$, and $g$ are treated by Hauk as appropriate constants.

Hauk considered the problem of the above equations subject to the (constant) initial conditions:

$$
\left.\begin{array}{l}
T(x, 0)=T_{0} \\
\Psi(x, 0)=0
\end{array}\right\}
$$

and boundary conditions corresponding to diffusion-lirrited moisture uptake by the atmosphere and a constant net heat flux at the ground surface:

$$
\left.\begin{array}{ll}
k \frac{\partial \psi}{\partial x}=A_{v}\left(\Psi-\psi_{d}\right), & \text { at } x=0 \\
-k \frac{\partial T}{\partial x}=R_{n}, & \text { at } x=0
\end{array}\right\}
$$

This problem was solved by Hauk using Laplace transforms with respect to the time variable. The solutions are expressed in terms of dimensionless ratios: 


$$
\begin{aligned}
& T_{1}=\left(T-T_{0}\right) / T_{0} \\
& \Psi_{1}=\psi / \Psi_{\mathrm{a}} \\
& \left.\xi=x / L_{1}\right) \\
& \left.\tau=t / t_{1}\right\} \quad L_{1}, t_{1} \text { arbitrary }
\end{aligned}
$$

The results are:

$$
\begin{aligned}
\Psi_{1}(\xi, \tau) & =\frac{1}{\left(a_{1}-b_{1}\right) \sqrt{a^{2}-b^{2}}}\left\{\left(H R_{a} a_{1} b_{1}\right) F_{2}(\sqrt{a+b} \xi, d, \tau)\right. \\
& -F_{2}(\sqrt{a-b} \xi, d, \tau) \\
& +a_{1}\left(R_{a} b_{1}+H\right) \sqrt{a-b} F_{1}(\sqrt{a+b} \xi, d, \tau) \\
& \left.-b_{1}\left(R_{a} a_{1}+H\right) \sqrt{a+b} F_{1}(\sqrt{a-b} \xi, d, \tau)\right\} \\
T_{1}(\xi, \tau) & =\frac{1}{\left(b_{1}-a_{1}\right) \sqrt{a}{ }^{2}-b^{2}}\left\{H R _ { a } \left[b_{1} F_{2}(\sqrt{a+b} \xi, d, \tau)\right.\right. \\
& \left.-a_{1} F_{2}(\sqrt{a-b} \xi, d, \tau)\right] \\
& +\left(R_{a} b_{1}+H\right) \sqrt{a-b} F_{1}(\sqrt{a+b} \xi, d, \tau) \\
& \left.-\left(R_{a} a_{1}+H\right) \sqrt{a+b} F_{1}(\sqrt{a-b} \xi, d, \tau)\right\}
\end{aligned}
$$

Here, the functions $F_{1}$ and $F_{2}$ are given by:

$$
F_{1}(x, d, t)=\frac{1}{d}\left\{\operatorname{erfc}\left(\frac{x}{2 \sqrt{t}}\right)-e^{d x+d^{2} t} \operatorname{erfc}\left(\frac{x}{2 \sqrt{t}}+d \sqrt{t}\right)\right\}
$$




$$
\begin{aligned}
F_{2}(x, d, t) & =\left\{\frac{2}{d} \sqrt{\frac{t}{\pi}} e^{-x^{2} / 4 t}-\frac{(1+d x)}{d^{2}} \operatorname{erfc}\left(\frac{x}{2 \sqrt{t}}\right)\right. \\
& \left.+\frac{1}{d^{2}} e^{x d+d^{2} t} \operatorname{erfc}\left(\frac{x}{2 \sqrt{t}}+d \sqrt{t}\right)\right\}
\end{aligned}
$$

The various constants appearing in Equations (4.6 through 4.9 ) are given by:

$$
\begin{aligned}
& a_{1}=\frac{k(a+b)-1}{k \pi(a+b)} \\
& b_{1}=\frac{k(a-b)-1}{k \pi(a-b)} \\
& a=\frac{\lambda+k}{2 \lambda k(1-\delta \pi)} \\
& b=\left[a^{2}-\frac{1}{\lambda \kappa(1-\delta \pi)}\right]^{1 / 2} \\
& d=H \frac{a_{1} \sqrt{a-b}-b_{1} \sqrt{a+b}}{\left(a_{1}-b_{1}\right) \sqrt{a^{2}-b^{2}}} \\
& k=K_{a} t_{1} / C L_{1}^{2} \\
& \pi=\frac{w \Psi_{a}}{K_{a} \bar{T}_{0}} \\
& s=\frac{V T_{0}}{K_{a} \Psi_{a}} \\
& \lambda=\frac{k_{a} t_{1}}{C_{m} L_{1}^{2}} \\
& g^{\prime}=g L / \Psi_{a} \\
& H=A_{v} L / K \\
& R_{a}=R_{n} L / K T_{0}
\end{aligned}
$$


An alternative model for coupled moisture and heat flow was suggested by Nicholaichuk (1974) who began with rather general expressions for the moisture flux due to Phi\}ip and devries (1957):

$$
q_{\theta}=-K \frac{\partial}{\partial x}(\Psi-x)-D_{T} \frac{\partial T}{\partial x}
$$

This expression is linear in the gradients of moisture potential, gravitational potential, and temperature. By defining the soil water diffusivity:

$$
D_{\theta}=k \frac{d \Psi}{d \theta} \quad\left(\mathrm{cm}^{2} / \mathrm{sec}\right)
$$

this expression takes the more symmetrical form:

$$
q_{\theta}=K-D_{\theta} \frac{\partial \theta}{\partial x}-D_{T} \frac{\partial T}{\partial x}
$$

The moisture flow equation then becomes:

$$
\frac{\partial \theta}{\partial t}=-\frac{\partial}{\partial x} q_{\theta}=\frac{-\partial K}{\partial x}+\frac{\partial}{\partial x}\left(D_{\theta} \frac{\partial \theta}{\partial x}\right)+\frac{\partial}{\partial x}\left(D_{T} \frac{\partial T}{\partial x}\right)
$$

Treating $K$ as a function of $T$ and $\theta$, and defining:

$$
\left.\begin{array}{l}
M=\frac{\partial K}{\partial \theta} \\
N=\frac{\partial K}{\partial T}
\end{array}\right\}
$$

then leads to:

$$
\frac{\partial \theta}{\partial t}=\frac{\partial}{\partial x}\left(D_{\theta} \frac{\partial \theta}{\partial x}\right)+\frac{\partial}{\partial x}\left(D_{T} \frac{\partial T}{\partial x}\right)-M \frac{\partial \theta}{\partial x}-N \frac{\partial T}{\partial x}
$$


The coefficients $D_{\theta}, D_{T}, M$ and $N$ are, in general, functions of $T$ and $\theta$. For purposes of obtaining an analytical solution, Nichoiaichuk treated them as constants.

A corresponding equation for $\partial T / \partial t$ was obtained by Nicholaichuk in somewhat less satisfying way. By expressing the heat flux $q_{h}$ as a linear combination of thermal conduction, vapor convection and liquid convection, he was lead to an equation for $\partial T / \partial t$ in which a number of highly nonlinear terms occur, e.g., $\partial \theta / \partial x \partial T / \partial x, T \partial \theta / \partial t$, etc. In order to obtain an equation tractable for analytical solutions, he simply ignored the troublesome terms, setting them to zero. It is not clear whether the resulting equation contains all the essential physics, but it has the simple form:

$$
\frac{\partial T}{\partial t}=F_{1} \frac{\partial^{2} T}{\partial x^{2}}-F_{4} \frac{\partial T}{\partial t}
$$

Here, $F_{1}$ and $F_{4}$ are appropriate constants.

Nicholaichuk suggested boundary conditions of the form:

$$
\left.\begin{array}{l}
\frac{\partial T}{\partial x}+K_{2} T=K_{1} \\
\frac{\partial \theta}{\partial x}=K_{3}+K_{4} T
\end{array}\right\} \quad \text { at } x=0
$$

It was later suggested by Gibos (1981) that a more consistent model is obtained with the boundary conditions:

$$
\left.\begin{array}{l}
\frac{\partial T}{\partial x}+K_{2} T=K_{1} \\
\frac{\partial \theta}{\partial x}-\frac{M}{D_{\theta}} \theta=K_{3}+K_{4} T
\end{array}\right\} \text { at } x=0
$$


Finally, initial conditions:

$$
\left.\begin{array}{l}
T=T_{0} \\
\theta=\theta_{0}
\end{array}\right\} \quad \text { at } t=0
$$

were proposed.

Nicholaichuk obtained analytical solutions of the above problem, using Laplace transforms with respect to $t$, first soiving Equation (4.6) for $T$, then using that result in Equation (4.15) and solving it for $\theta$. His results have never been published in a journal, but are available as a thes is (Nicholaichuk (1974). Unfortunately, the thes is appears to contain a very large number of typographical errors, making it an unreliable source of mathematical results. Because of this, the results were rederived by Gibbs (1981), including the case of the alternative boundary condition, Equation (4.18). These results are given in the report RHO-LP-48 (1981). The expression for $T$ is:

$$
\frac{T-T_{0}}{T_{0}}=\left(\frac{K_{1}}{a T_{0}}-\frac{K_{2}}{a}\right) g_{0}\left(x_{0}, t_{0}, R_{1}\right)
$$

Here:

$$
\begin{aligned}
a & =F_{4} / 2 F_{1} \\
x_{0} & =a x \\
t_{0} & =t / \tau \\
R_{1} & =-K_{2} / \alpha \\
\tau & =1 / a^{2} F_{1}
\end{aligned}
$$

and:

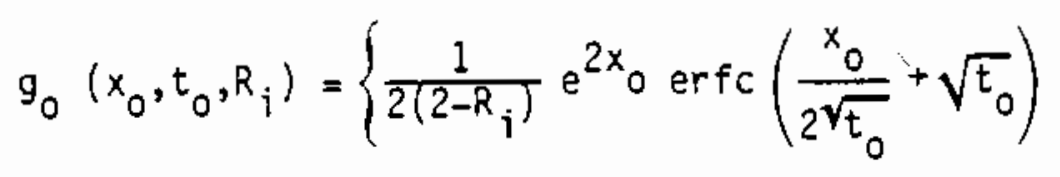




$$
\begin{aligned}
& -\frac{1}{2 R_{i}} \operatorname{erfc}\left(\frac{x_{0}}{2 \sqrt{t_{0}}}-\sqrt{t_{0}}\right) \\
& -\frac{\left(1-R_{j}\right)}{R_{j}\left(R_{i}-2\right)} e^{R_{i}\left(R_{i}-2\right) t_{0}+R_{i} x_{0}} \\
& \left.\operatorname{erfc}\left(\frac{x_{0}}{2 \sqrt{t_{0}}}+\left(R_{i}-1\right) \sqrt{t_{0}}\right)\right\}
\end{aligned}
$$

The expression for $\theta$ is rather lengthly:

$$
\theta=\theta_{0}+v_{p}\left(x_{0}, t_{0}\right)+v_{h}\left(x_{0}, t_{0}\right)
$$

Here:

$$
v_{p}\left(x_{0}, t_{0}\right)=C_{1} \sum_{i=1}^{2} B_{i} g_{0}\left(x_{0}, t_{0}, R_{i}\right)
$$

with:

$$
\begin{aligned}
& C_{1}=\left(\frac{K_{1}}{\alpha T_{0}}-\frac{K_{2}}{\alpha}\right) \\
& B_{1}=\frac{P_{1}-P_{2} R_{1}}{R_{1}-R_{2}} \\
& B_{2}=-B_{1}-P_{2} \\
& P_{1}=N_{\alpha} T_{0}^{\tau} \\
& P_{2}=O_{T} T_{0} \tau a^{2} \\
& R_{2}=\frac{M_{\alpha \tau}-2}{\tau D_{\theta}{ }^{2}-1}
\end{aligned}
$$


Also:

$$
v_{h}\left(x_{0}, t_{0}\right)=c_{1} f_{1}\left(x_{0}, t_{0}\right)+f_{2}\left(x_{0}, t_{0}\right)
$$

where:

$$
\begin{aligned}
f_{1}\left(x_{0}, t_{0}\right)= & \left\{\left(\frac{x_{0}}{2}+\frac{1}{4 \beta}+\frac{\beta \tau}{\eta} t_{0}\right) e^{2 \beta x_{0}}\right. \\
& \operatorname{erfc}\left(\frac{1}{2} \sqrt{\frac{\eta}{\tau}} \frac{x_{0}}{\sqrt{t_{0}}}+\beta \sqrt{\frac{\tau}{n}} \sqrt{t_{0}}\right) \\
& -\frac{1}{4 \beta} \operatorname{erfc}\left(\frac{1}{2} \sqrt{\frac{\eta}{\tau}} \frac{x_{0}}{\sqrt{t_{0}}}-\beta \sqrt{\frac{\tau}{\pi}} \sqrt{t_{0}}\right) \\
& \left.-\sqrt{\frac{\tau}{\pi \beta}} \frac{1}{\sqrt{t_{0}}} \exp \left(\beta x_{0}\right)-\left(\frac{\eta}{4} \frac{x_{0}^{2}}{t_{0}}+\frac{\beta^{2} \tau}{\eta} t_{0}\right)\right\}
\end{aligned}
$$

with:

$$
\left.\begin{array}{l}
\beta=M / 2 \alpha D_{\theta} \\
n=1 / \alpha{ }^{2} D_{\theta}
\end{array}\right\}
$$

Also:

$$
f_{2}\left(x_{0}, t_{0}\right)=\int_{0}^{t} h_{10}\left(t_{0}^{\prime}\right) h_{20}\left(x_{0}, t_{0}-t_{0}^{\prime}\right) d t_{0}^{\prime}
$$


with:

$$
\left.\begin{array}{l}
h_{10}\left(t_{0}\right)=-\frac{\tau}{n} \sum_{i=1}^{2} d_{i} g_{0}\left(0, t_{0}, R_{j}\right) \\
d_{1}=\frac{K_{4}}{a^{2}}\left(K_{1}-K_{2} T_{0}\right)-C_{1} 8_{1}\left(R_{1}-2 \beta\right) \\
d_{2}=-C_{1} B_{2}\left(R_{2}-2 \beta\right)
\end{array}\right\}
$$

and:

$$
\begin{aligned}
h_{2}\left(x_{0}, t_{0}\right) & =\left\{\sqrt{\frac{\pi}{\pi \tau}} \frac{1}{\sqrt{t_{0}}} \exp -\left(\frac{1}{2} \sqrt{\frac{\pi}{\tau}} \frac{x_{0}}{\sqrt{t_{0}}}-\beta \sqrt{\frac{\pi}{\pi}} \sqrt{t_{0}}\right)^{2}\right. \\
& \left.-\beta e^{2 \beta x_{0}} \operatorname{erfc}\left(\frac{1}{2} \sqrt{\frac{\pi}{\tau}} \frac{x_{0}}{\sqrt{t_{0}}}+\beta \sqrt{\frac{\pi}{\pi}} \sqrt{t_{0}}\right)^{2}\right\}
\end{aligned}
$$

A computer program "MOIST" has been written by Gibbs (1980) to evaluate the above expressions. Limited comparisons with finite element numerical solutions of the same equations have been reported (1980). 


\subsection{NUMERICAL EVALUATION OF SPECIAL FUNCTIONS}

Analytical solutions of moisture flow problems often involve certain special functions such as Bessel functions, exponential integrals, or error functions. Highly accurate numerical methods exist for evaluating these functions. Modern routines can be obtained from the Argonne National Laboratory FUNPACK Library, the International Mathematical and Statistical Library (IMSL) avajlable on the BCSR UNIVAC 1100 and the Batteile OEC VAX-11, or from specific papers in the literature. These routines are usually based on rational approximations, i.e., expressions for the desired special function as a quotient of polynomials. Often, different polynomials are used, depending on whether the argument of the function is large or small. A slightly less efficient, but equally accurate, approach is to use the series expansions, continued fractions, and asymptotic expansions given, e.g., in the NBS Handbook of Mathematical Functions, AMS-55 (1964). Either of the above approaches leads to accurate numerical values for the common special functions.

It is easy to assume that having accurate values of special functions will guarantee an accurate evaluation of analytical solutions of moisture flow probiems. This attitude can be overly optimistic, since analytical solutions often involve lengthly expressions containing differences or products of special functions. Round-off errors can be large, and products of large numbers with small numbers can easily degenerate, in a computational environment, to the indeterminate form "underflow $x$ overflow."

The above problems can be particularly pronounced when combinations of exponentials and error functions are involved. For example, the function:

$$
f(x, y)=e^{\alpha x} \operatorname{erfc}(\beta y)
$$

easily reduces to $\infty x O$ if $a x$ and $B y$ are both very large. However, for large arguments:

$$
\operatorname{erfc}(\beta y)=\frac{1}{\sqrt{\pi}} e^{-\beta^{2} y^{2}}\left(\frac{1}{x+\frac{1}{2 x+\ldots}}\right)
$$


and thus:

$$
f(x, y)=\frac{1}{\sqrt{\pi}} e^{\alpha x-\beta y^{2}}\left(\frac{1}{x+\frac{1}{2 x+\ldots}}\right)
$$

By combining the exponents before calling the exp routine the problem of $\infty \times 0$ is avoided.

Similarly, the function:

$$
g(x, y, z)=e^{x}(\text { erf } y \rightarrow \text { erf } z)
$$

can reduce to $\infty \mathrm{x} 0$ if all three arguments are very large. In addition, considerable round-off error can occur in evaluating the difference of the erf's if $y$ and $z$ both exceed about 2.0. Here, a possible approach is to use the definition:

$$
\operatorname{erf} y=\frac{2}{\sqrt{\pi}} \int_{0}^{y} e^{-t^{2}} d t
$$

to write:

$$
\begin{aligned}
\operatorname{erf} y-\operatorname{erf} z & =\frac{2}{\sqrt{\pi}} \int_{z}^{y} e^{-t^{2}} d t \\
& =\frac{2}{\sqrt{\pi}} e^{-z^{2}} \int_{0}^{y-z} e^{-\tau^{2}-2 z \tau} d \tau
\end{aligned}
$$

where the change of variable $\tau=t-z$ has been used. The exponential in the last integral is the generating function for the Hermjte polynomials (Sneddon 1956), i.e.: 


$$
e^{-\tau^{2}-2 z \tau}=\sum_{n=0}^{\infty} \frac{(-1)^{n}}{n !} H_{n}(z) \tau^{n}
$$

Thus:

$$
\text { erf } y-\operatorname{erf} z=\frac{2}{\sqrt{\pi}} e^{-z^{2}} \sum_{n=0}^{\infty} \frac{(-1)^{n}}{n !} H_{n}(z) \int_{0}^{y-z} \tau^{n} d t
$$

The integrals are elementary, giving:

$$
\text { erf } y-\operatorname{erf} z=\frac{2}{\sqrt{\pi}} e^{-z^{2}} \sum_{n=0}^{\infty} \frac{(-1)^{n_{H}}(x)}{(n+1) !}(y-z)^{n+1}
$$

Thus, multiplying by $\mathrm{e}^{\mathrm{x}}$ gives:

$$
g(x, y, z)=\frac{2}{\sqrt{\pi}} e^{x-z^{2}} \sum_{n=0}^{\infty} \frac{(-1)^{n_{H}}(x)}{(n+1) !}(y-z)^{n+1}
$$

If $y-z$ is not too large, the series may converge rapidily requiring only a few terms. Even if $x, y$ and $z$ are all large, the problem of loss of significant figures is minimized by computing the difference $y-z$ rather than erf $y-$ erf $z$, and the problem of $\infty x 0$ is minimized by combining the $x-z^{2}$ terms before calling the exp routine.

In the above example, if the difference $y-z$ is too large, then it is better to use the expansion:

$$
\text { erf } y=1-\operatorname{erfc} y=\frac{1}{\sqrt{\pi}} e^{-y^{2}}\left(\frac{1}{y+\frac{1}{2 y+\ldots}}\right)
$$

and similarly for erf $z$, to obtain: 


$$
\begin{aligned}
& g(x, y, z)=\frac{1}{\sqrt{\pi}}\left\{e^{x-y^{2}}\left(\frac{1}{y+\frac{1}{2 y+\ldots}}\right)\right. \\
& -e^{x-z^{2}}\left(\frac{1}{z+\frac{1}{2 z+\ldots}}\right)
\end{aligned}
$$

Clearly, depending on the values of $x, y$, and $z$, the function $g(x, y, z)$ may be evaluated either directly using Equation (5.4) or by using the Hermite expansion as in Equation (5.10), or by using the asymptotic expansion of Equation (5.12).

The above example should indicate that the evaluation of expressions involving certain special functions can be demanding, even when the individual functions are available to high accuracy. The development and testing of a function routine to evaluate a function, such as $g(x, y, z)$ in the example above, for all conceivable values of the arguments can be challenging and time-consuming. If the particular application requires only arguments in a certain 3 imited range, then considerable time may be saved by developing a method which works well for that range. The only pitfall is that the original limitations must be remembered if the same routine is to be used in a later application.

As illustrations of how some of the above special functions and their combinations may be computed, FORTRAN listings of programs to compute erf $(x)$, $\operatorname{erfc}(x), e^{x} \operatorname{erfc}(y)$, and $e^{x}[\operatorname{erf}(y)-\operatorname{erf}(z)]$ appear in Appendix $I$. 


\subsection{EXTENSIONS OF ANALYTICAL SOLUTIONS BY PERTURBATION THEORY}

As noted above, analytical solutions of the moisture flow equations are obtained only with simplifying assumptions, such as that certain coefficients in the equations are constants, or at least are very elementary functions.

These solutions often can be extended, using perturbation theory, to obtain solutions of problems whose coefficients are not elementary, but which differ only by small quantities from being elementary. Of course, just as there are functions in calculus which do not have a derivative, or which have discontinuous derivatives, so too there are physical problems in which a small change in the equations leads to a large change in the solutions. These cases cannot be treated by elementary perturbation theory. But in many physical problems, small changes in the equations do lead to smail changes in the solutions, and the treatment of these is summarized here (Lewins 1965 and Cacuci et al. 1980).

Suppose that an analytical solution has been obtained to an idealized linear moisture flow problem whose basic differential equation is abbreviated as:

$$
L \theta=S
$$

Here, $L$ denotes the linear differential operator and $S$ denotes any source terms. The symbol $\theta$ denotes the dependent variable (either $\theta, \Psi(\theta), \phi(\Psi)$, etc).

Now, consider a problem with variable coefficients which is "close" to this problem, i.e., we replace the operator $L$ by $L+\delta L$, where $\delta L$ denotes the (smal1) change in the operator. For generality, suppose that the source $S$ is also replaced by $S+\delta S$. Because of the perturbations in $L$ and $S$, the solution $\theta$ is replaced by $\theta^{+} \delta \theta$, where:

$$
(L+\delta L)\left(\theta^{+} \delta \theta\right)=S+\delta S
$$


If $L+\delta L$ is aiso linear then:

$$
L \theta+L \delta \theta+\delta L \theta+\delta L \delta \theta=S+\delta S
$$

Since the quantities involving s are small, we make the approximation that $\delta L \delta \theta$ can be neglected as a second order term. Then subtracting (6.1) from (6.3) yields:

$$
L \delta \theta \simeq \delta S-\delta L \theta
$$

Thus, formally:

$$
\delta \theta=L^{-1}(\delta S-\delta L \theta)
$$

Here, $L^{-1}$ is the inverse of the differential operator for which we have the analytical solution, and it is understood that se satisfies apropriate boundary or initial conditions. (Actually, we don't need to calculate $L^{-1}$; see below!)

Suppose now that some particular result of the calculation is needed; one which can be written as an integral over the solution, i.e., the result $R$ has the form:

$$
R=\int S^{+}(x) \theta(x) d x
$$

Here, $S^{+}(x)$ denotes some weight function, and $d x$ is shorthand notation for integrating over all the independent variables, i.e., " $\int \mathrm{dx}$ " $=\int \mathrm{dx} \int \mathrm{dy} \int \mathrm{dt}$, or whatever your problem has in it. If the result of interest is the value of $\theta$ at a particular "point" $x=x_{0}$, then take $S^{+}(x)=\delta\left(x-x_{0}\right)$ so that:

$$
R \rightarrow \int \delta\left(x-x_{0}\right) \theta(x) d x=\theta\left(x_{0}\right)
$$

If the result is the total moisture in a subregion $G$, then $S^{+}=1$ if $x_{\varepsilon} G$ and $S^{+}=0$ if $x \notin G$, i.e., then: 


$$
R \rightarrow \int_{E} G \theta(x) d x
$$

(These examples are meant to show that a variety of types of results can be written in the form (6.5) by an appropriate choice of the function $S^{+}(x)$.)

Now, if $R$ is obtained from the analytical solution of the original problem (6.1), and if the perturbed problem is considered, then $R$ is replaced by:

$$
R+\delta R=\int S^{+}(x)[\theta(x)+\delta \theta(x)] d x
$$

or, subtracting (6.5), the change in $\mathrm{R}$ is:

$$
\delta R=\int S^{+}(x) \delta \theta(x) d x
$$

Substituting $\delta \theta$ from $(6.4)$ gives:

$$
\delta R=\int S^{+}(x) L^{-1}[\delta S(x)-\delta L \theta(x)] d x
$$

Now, recall the definition of the adjoint operator $L^{+}$: if $f(x)$ and $g(x)$ are any two functions in the proper domains, then $L^{+}$is defined so that:

$$
\int f(x) \operatorname{Lg}(x) d x=\int\left[L^{+} f(x)\right] g(x) d x
$$

Now, let $\theta^{+}(x)$ be a function satisfying the equation:

$$
L^{+} \theta^{+}(x)=S^{+}(x)
$$

Then, Equation (6.10) becomes:

$$
\delta R=\int\left[L^{+} \theta^{+}(x)\right] L^{-1}[\delta S(x)-\delta L \theta(x)] d x
$$

By the property of the adjoint operator, Equation $(6.11)$, this can be written as: 


$$
\begin{aligned}
\delta R & =\int \theta^{+}(x) L L^{-1}[\delta S(x)-\delta L \theta(x)] d x \\
& + \text { "boundary terms" }
\end{aligned}
$$

Since $L^{-1}=I$, the operators cancel. The "boundary terms" arise since going from (6.13) to (6.14) usually requires integration by parts, Green's theorem, et al. The trick is to pick the boundary and injtial conditions on $\theta^{+}(x)$ to make the "boundary terms" vanish. (It's usually obvious how to do this in any particular case.) Suppose this has been done. Then, Equation (6.14) reduces to:

$$
\delta R=\int_{\theta}^{+}(x)[\delta S(x)-\delta L \theta(x)] d x
$$

If, in particular, $S^{+}(x)$ had been chosen to be $\delta\left(x-x_{0}\right)$, then $R$ is $\theta\left(x_{0}\right)$ and $\delta R$ would represent the change in $\theta$ at $x_{0}$ due to the perturbations $\delta L$ and $\delta$.

Let's summarize how this expression can be used:

1. An analytical solution is obtained to the simple problem $L \theta=S$.

2. The operator $L^{+}$is determined from the identity $\int_{\theta}^{+} \operatorname{Lodx}=$ $\int\left(\mathrm{L}^{+} \theta^{+}\right) \theta d x$, and boundary and initial conditions for $\theta^{+}$are chosen to get rid of any "boundary terms" that appear here.

3. The adjoint equation $\mathrm{L}^{+} \theta^{+}=S^{+}$must be solved, subject to these conditions. This is usualiy the same degree of difficulty as the original equation $L \theta=S$; thus, this can probably be done analytically too.

4. Now, any problem which is "close" to the original problem (i.e., it differs by a small $\delta S$ and $\delta L$ ) will have a solution $\theta^{+} \delta \theta$, leading to a change in the result $R$ by an amount $\delta R$ given by Equation $(6.15)$. It may be possible to do the integral in closed form, but at least everything under the integral is known.

Note: this is an approximate method, since second order terms $\delta$ L $\delta \theta$ were ignored. But if the $\delta$ terms are, say, $10 \%$ of the original terms, then $\delta^{2}$ may be $=1 \%$, which may be negligible. 


\section{REFERENCES}

Abramowitz M., and I. A. Stegun. 1964. Handbook of Mathematical Functions, U.S. National Bureau of Standards, App 1. Math. Ser., Vol. 55, U.S.G.P.0., Washington.

Cacuci, D. G. et al. 1980. "Sensitivity Theory for General Systems of Nonlinear Equations." Nucl. Sci. Engng. 75:88.

Carslaw, H. S., and J. C. Jaeger. 1948. Conduction of Heat in Solids, Oxford, London.

Gibbs, A. G., and R. G. Baca. 1981. Analytical Models for Simultaneous Heat and Moisture Transport in Soils, RHO-LD-48, Rockwell Hanford Co.

Hansen, A. G. 1964. Similarity Analyses of Boundary Value Problems in Engineering. Prentice-Hall, Englewood Cliffs.

Hauk, R. W. 1971. Soil Temperature and Moisture Fields Predicted by a System of Coupled Equations, Thesis, Cornell University, Ithaca, New York.

Lewins, J. 1965. Importance, the Adjoint Function, Pergamon, New York.

Lomen, D. 0., and A. W. Warrick. 1974. "Time-Dependent Linearized

Infiltration: II. Line Sources." Soil Sci. Soc. Amer. Proc. 38:568.

Lomen, D. 0., and A. W. Warrick. 1978. "Time-Dependent Solutions to the OneDimensional Linearized Moisture Flow Equation with Water Extraction." $\mathrm{J}$. Hydrology. 39:59.

Nicholaichuk, W. 1974. A Soil Moisture and Temperature Prediction Model Under Evaporative Conditions, Thes is, University of Guelph, Guelph, Ontario.

Philip, J.R. 1966. "A Linearization Technique for the Study of Infiltration." Proc. UNESCO Netherlands Symp. on Water in the Unsaturated Zone, pp. 471-478.

Philip, J. R. 1969. "Theory of Infiltration." In Advances in Hydroscience, $5: 215-305$.

Philip, J. R., and D. A. Devries. 1957. "Moisture Movement in Porous Materials Under Temperature Gradients." Trans. Amer. Geophys. Union. $38: 222$.

Rainville, E. D. 1958. Elementary Differential Equations, MacMillan, New York.

Sneddon, I. N. 1956. Special Functions of Mathematical Physics and Chemistry, $0 l i v e r$ and Boyd, Edinburgh. 
Tranter, C. J. 1974. Integral Transforms in Mathematical Physics, Chapman and Hall, London.

Warrick, A. W. 1974. "Time Dependent Linearized Infiltration. I. Point Sources." Soil Sci. Soc. Amer. Proc. 38:383.

Warrick, A. W., and D. O. Lomen. 1976. "Time Dependent Linearized

Infiltration: III. Strip and Disk Source." Soil Sci. Soc. Amer. Proc. 40:639. 


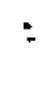




\section{APPENDIX I}

FORTRAN PROGRAMS FOR THE FUNCTIONS 
APPENDIX I

\title{
FORTRAN PROGRAMS FOR THE FUNCTIONS
}

\author{
(A. G. Gibbs) \\ $\operatorname{erf}(x)$ \\ $\operatorname{erfc}(x)$ \\ $e^{x} \operatorname{erfc}(y)$ \\ $e^{x}[\operatorname{erf}(y)-\operatorname{erf}(z)]$
}

A. 1 


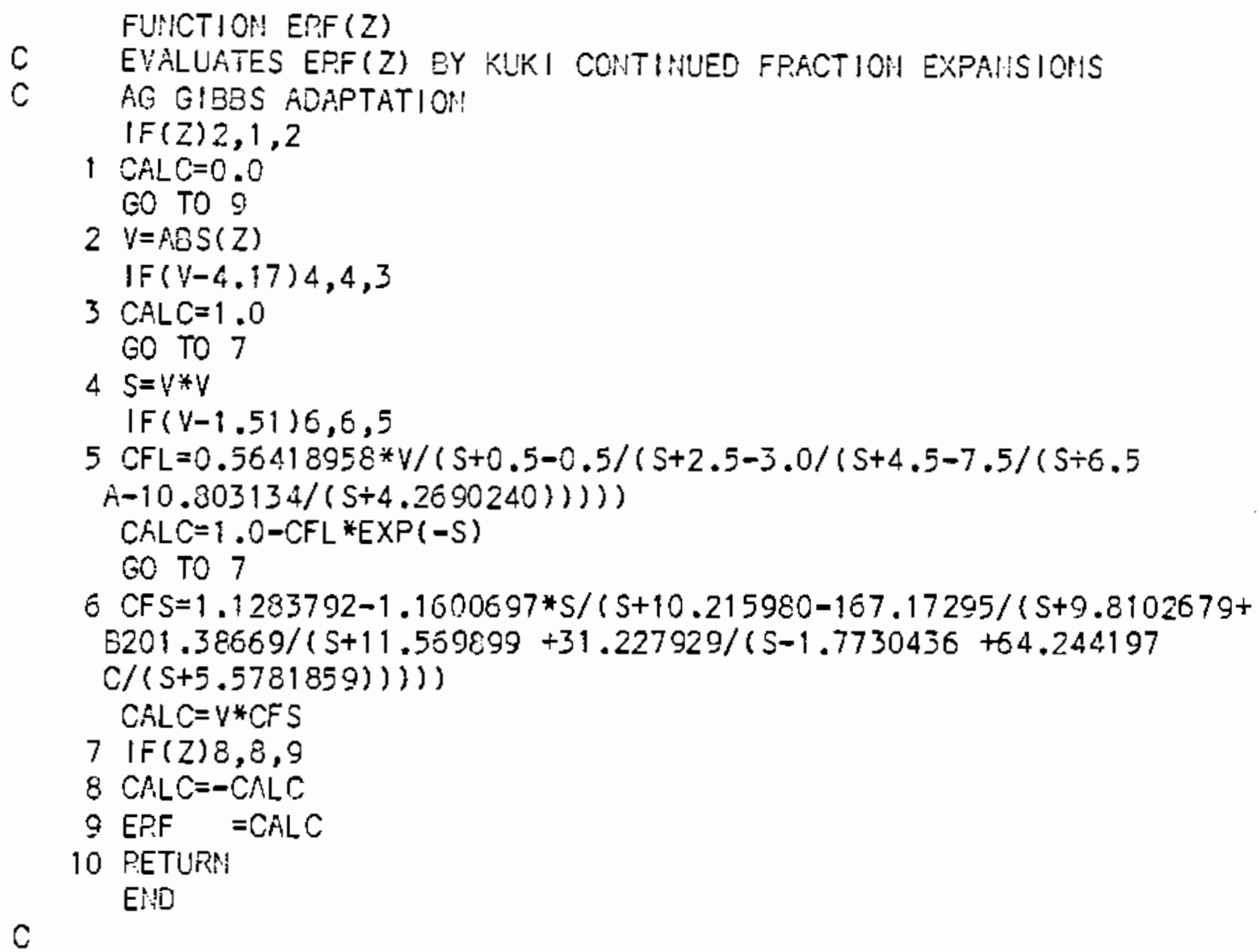




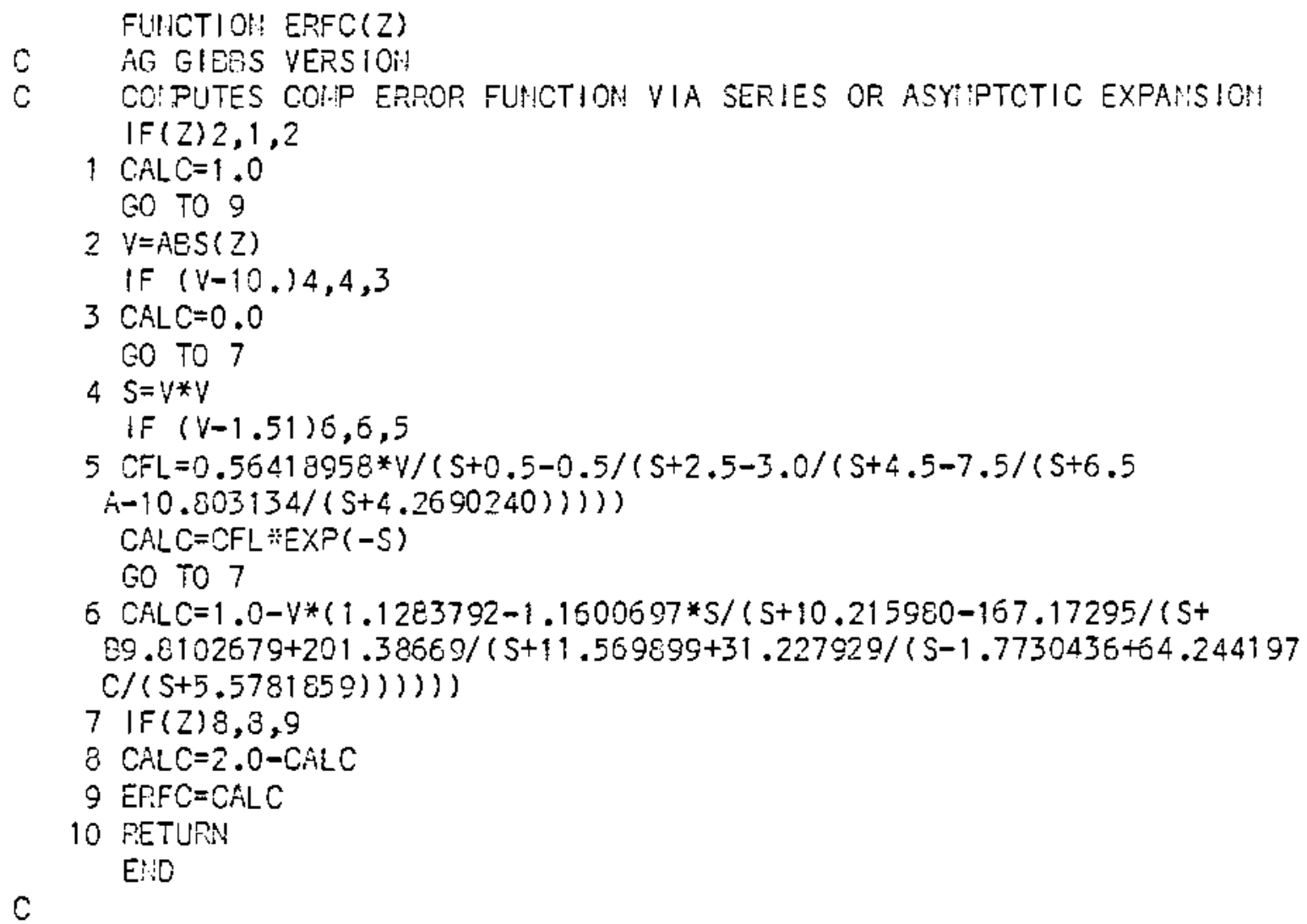

A. 3 


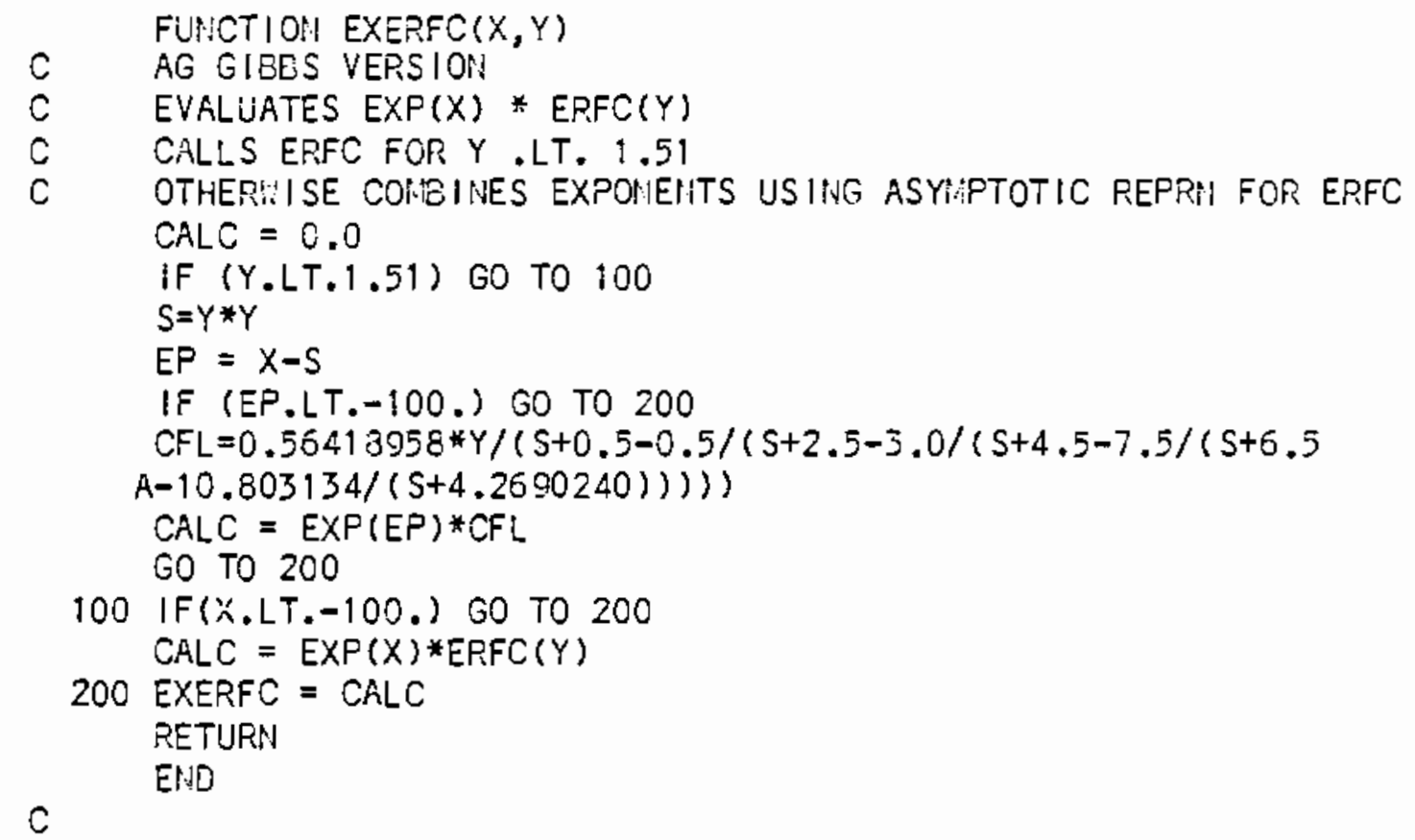




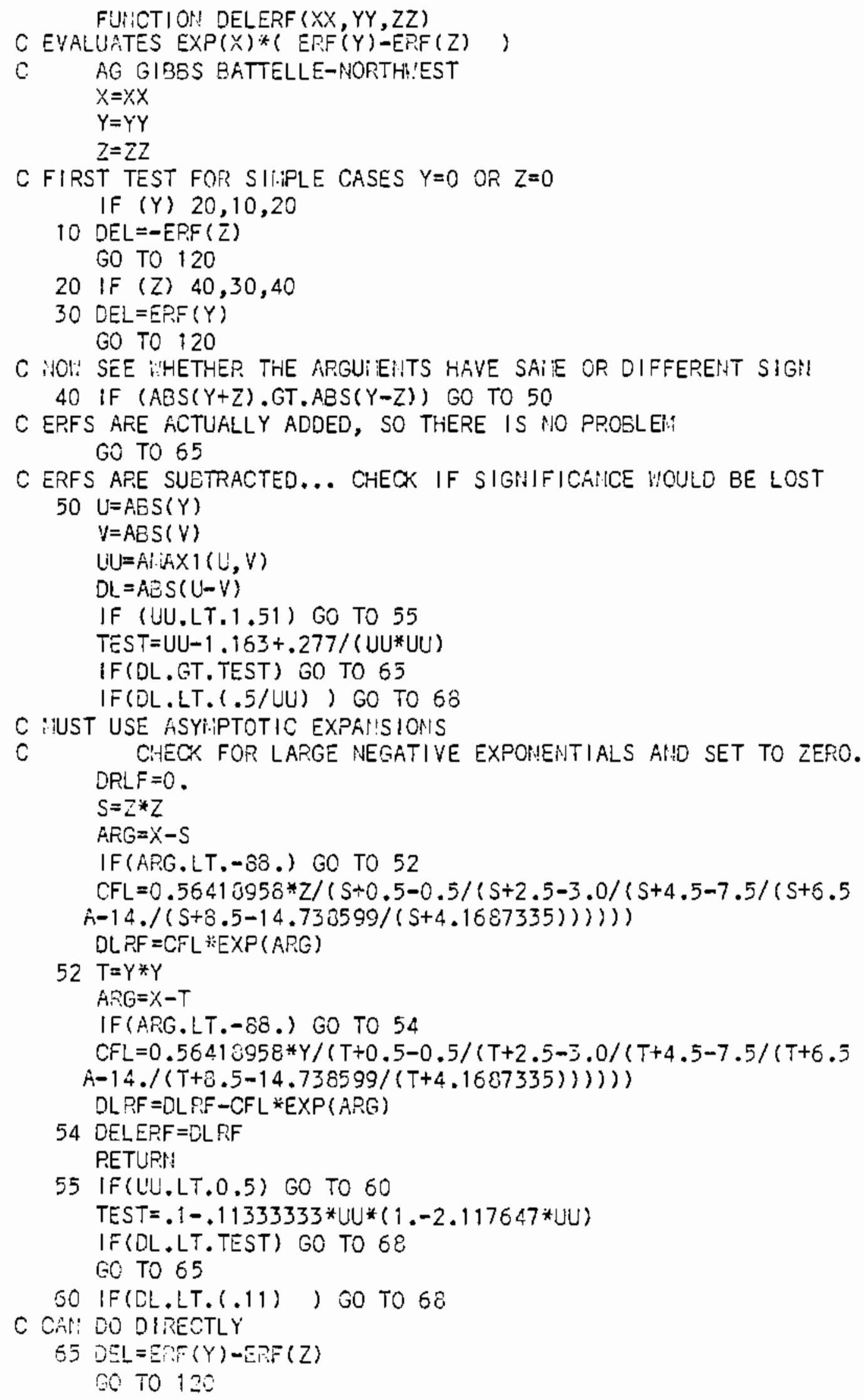




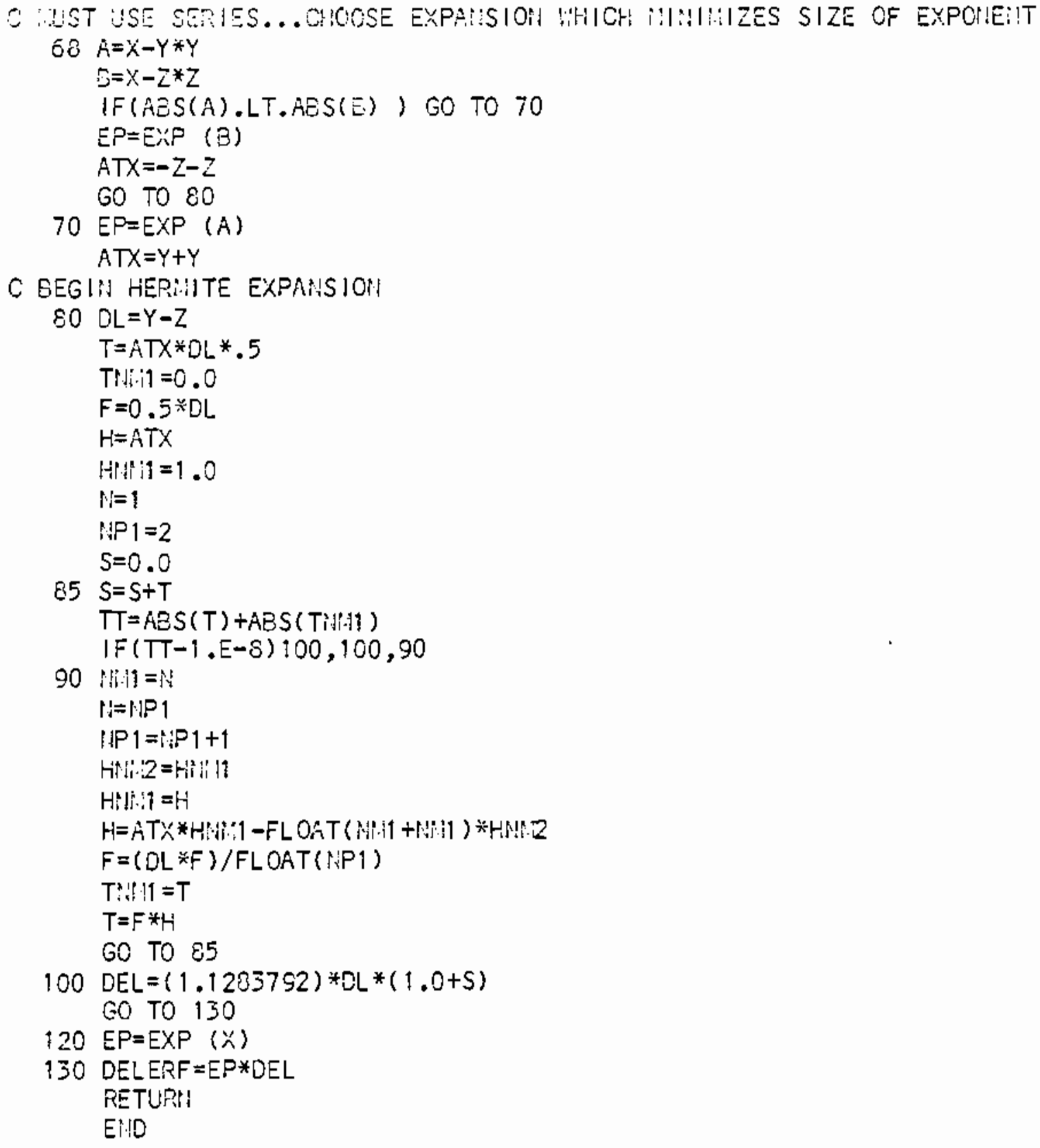




\section{DISTRIBUTION}

No. of

Copies

\section{OFFSITE}

27 DOE Technical Information Center

R. E. Cunningham

Office of Nuclear Safety

Materials and Safeguards

Room 562

Nuclear Regulatory Commission

7915 Eastern Avenue

Silver Springs, MD 20910

3 Division of Waste Management Nuclear Regulatory Commission Washington, D.C. 20555

ATTN:
J. B. Martin
D. B. Rohrer
R. D. Smith

W. E. Mott

DOE Division of Environmental Control Technology

Washington, D.C. 20545

4 DOE Office of Nuclear Waste

Management

NEW, B-107, HQ

Washington, D.C. 20545

ATTN: G. Oertel

J. D. Dieckhoner

D. J. McGoff

C. A. Heath

S. A. Mann

DOE Chicago Operations and Region Office

Argonne, IL 60439
No. of

Copies

J. 0. Neff

DOE Columbus Program Office

$505 \mathrm{King}$ Avenue

Columbus, $\mathrm{OH} 43201$

3 DOE Idaho Operations Office

505 Second Street

Idaho Fal1s, ID 83401

ATTN: J. P. Hamric

J. B. Whitsett

J. W. Pee?

3 DOE Oak Ridge Operations Office

P. 0. Box E

Oak Ridge, TN 37830

ATTN: S. W. Ahrends (2)

D. E. Large

3 DOE Albuquerque Operations Office

P. 0. Box 5400

Albuquerque, NM 87185

ATTN: R. Y. Lowrey (2)

A. L. Taboas

S. G. Harbinson

DOE San Francisco Operations Dffice

1333 Broadway

Oakland, CA 94612

2 Environmental Protection Agency

Technological Assessment Division (AW-559)

Office of Radiation Programs

Washington, D.C. 20460 
No. of

Copies

3 Washington State University

Pullman, WA 99164

ATTN: Dr. W. H. Gardner

Dr. G. S. Campbell

Dr. A. Cass

11 Oregon State University

Corvalis, OR

ATTN: Dr. S. W. Childs

Dr. A. G. Gibbs (10)

New Mexico State University Las Cruces, NM

ATTN: Dr. P. J. Wierenga

2 Battelle Memorial Institute

$505 \mathrm{King}$ Avenue

Columbus, $\mathrm{OH} 43201$

ATTN: Beverly Rawles

Research Library

3 EG\&G Idaho

P. 0. Box 1625

Idaho Falls, ID 83415

ATTN: M. D. McCormack

G. B. Levin

M. L. Krupinski

Robert Williams

Electric Power Research

Institute

3412 Hjllview Avenue

Palo Alto, CA 94304

3 Oak Ridge National Laboratory

P. 0. Box $X$

Oak Ridge, TN 37830

ATTN: Central Research Library

A. L. Lotts

R. S. Lowrie
No. of

Copies

ONSITE

4 DOE Richland Operations Office

P. A. Craig (2)

R. D. Gerton

H. E. Ransom

2 Rockwell Hanford Operations

J. B. Sisson

C. W. Manry

49 Pacific Northwest Laboratory

R. L. Brodzinski

R. A. Brouns/W. F. Bonner

T. D. Chikalla

J. A. Franz

G. W. Gee (5)

M. S. Hanson/M. J. Schliebe

A. E. Jaquish

T. L. Jones (10)

J. M. Latkovich

L. Y. Martin

H. L. Nielson

A. M. Platt

J. V. Robinson

R. I. Smith/E. S. Murphy

S. L. Stein

J. L. Swanson

H. H. Van Tuyl/

$R$. E. Nightingale

R. A. Walter $/ D$. B. Cearlock

N. A. Wogman/J. M. Nielson

Technical Information (5)

Publishing Coordination BE (2)

Water \& Land Research

Library (10) 